\title{
Partielle Thromboplastinzeit, Aktivitäten von Faktor VIII und Faktor IX bei klinisch unauffälligen Kindern im Vergleich mit gesunden Erwachsenen
}

\section{Partial Thromboplastin Time, Activities of Factor VIII and Factor IX in Children without Apparent Coagulation Disorders in Comparison to Normal Adults}

\author{
Gisela Wanner', Janny van der Woerd-de Lange', L. Weiss', N. Hegner ${ }^{2}$ \\ ${ }^{1}$ Institut für Klinische Chemie am Städt. Krankenhaus München Harlaching \\ 2 Behringwerke AG, Frankfurt/Main
}

\section{Zusammenfassung:}

Bei 92 klinisch unauffälligen Kindern im Alter von zwei bis zehn Jahren wurde die aktivierte Partielle Thromboplastinzeit (PTT) mit zwei verschiedenen Reagenzien gemessen und mit einem Kollektiv von 69 gesunden Erwachsenen verglichen.

Überprüft werden sollte, inwieweit die Richtwerte für Erwachsene auch für diese Altersgruppe gelten. Bei der Neothromtin ${ }^{\circledR}$-Methode fanden sich statistisch gesicherte Verlängerungen um fünf Sekunden gegenüber der 95. Percentile des Erwachsenenkollektivs (Erwachsene bis $38 \mathrm{sec}$, Kinder bis $43 \mathrm{sec}$ ). Bei der Actin ${ }^{\circledR}$-FS-Methode konnte nur im Frischplasma eine statistisct gesicherte Differenz zwischen Erwachsenen- und Kinderkollektiv festgestellt werden (Erwachsene bis $39 \mathrm{sec}$, Kinder bis $40 \mathrm{sec}$ ). Die möglichen Ursachen wurden diskutiert und auf die Notwendigkeit hingewiesen, eigene Richtwerte unter den in der Klinik üblichen Bedingungen sowohl für Erwachsene als auch für Kinder zu erstellen.

\section{Sch/üsselwörter:}

PTT - Kinder-Normalwerte - Faktor VIII - Faktor IX

\section{Summary:}

The aim of this study was to investigate whether the normal values of activated partial thromboplastin time for adults are also valid for children age two to ten. 92 children are compared with 69 adults applying two diffrerent reagents.

With Neothromtin ${ }^{\circledR}$ a significant prolongarion of five seconds were found ladults: upper level 38 sec, children: upper level $43 \mathrm{sec}$. With Actin ${ }^{\circledR}$-FS only in fresh plasma a statistically significant prolongation was obtained (adults upper level $39 \mathrm{sec}$, children: upper level $40 \mathrm{sec}$ ). The possible causes are discussed and it is pointed out that normal values of adults cannot be applied for pediatrics. Each clinical laboratory should determine its own reference values for adults and children.

Keywords: -.-

PTT - children normal values - factor VIII - factor IX

\section{Einleitung}

Die Bestimmung der Thromboplastinzeit nach Quick und der aktivierten partiellen Thromboplastinzeit.(PTT) gehört zum präoperativen Untersuchungsspektrum. Mit diesen Bestimmungen will man ernsthafte Gerinnungsstörungen rechtzeitig erfassen. Aus klinischer Sicht hat es den Anschein, daß bei Kindern (zwei- bis zehnjährig) die PTT öfter oberhalb des in der jeweiligen Klinịk üblichen Richtwertes liegt, ohne daß eine Gerinnungsstörung anamnestisch oder durch Einzelfaktorenanalyse nachgewiesen werden kann. Dabei soll es offensichtlich unabhängig sein, welche Technik, manuell oder automatisch oder welches PTT-Reagenz verwendet wird. Eine "verlängerte PTT" führt häufig zum Zurückstellen von Operationen, zu einer Reihe von Kontrollen und weiteren zeit- und kostenaufwendigen Untersuchungen.
Gängige Normalwerttabellen (1, 2) enthalten den Hinweis, daß Erwachsenen-Normalwerte für die PTT mit zwei bis neun Monaten erreicht werden. In einer sehr ausführlichen Literaturrecherche zurück bis zum Jahr 1973 konnten wir lediglich eine Kurzmitteilung über den Gehalt einiger Gerinnungsfaktoren bei Kindern verschiedenen Lebensalters (3), zwei Publikationen über Änderungen von Gerinnungsfaktoren unter Reduktionsdiät von neun- bis vierzehnjährigen Kindern $(4,5)$ und zwei Publikationen über Richtwerte bei Frühgeborenen und Säuglingen $(6,7)$ finden.

Untersuchungen über PTT-Normalwerte im Kleinkindund Schulalter liegen anscheinend nicht vor.

Sinn der vorliegenden Studie war es daher, zu überprüfen, inwieweit sich die gemessenen PTT-Werte von zweibis zehnjährigen Kindern von einer Kontrollgruppe acht- 
zehn- bis vierzigjähriger Erwachsener unterscheiden. In diesem Zusammenhang wurde auch die Aktivität der Faktoren VIII und IX mitgemessen.

\section{Material und Methoden}

Im Zeitraum von Januar 1989 bis Februar 1990 wurden 191 klinisch unauffällige Personen getestet, und zwar 114 Kinder im Alter von zwei bis zehn Jahren und 77 Erwachsene zwischen achtzehn und vierzig Jahren. Aufgenommen in die Studie wurden Kinder, die zu einem operativen Eingriff im entzündungsfreien Intervall zur stationären Aufnahme wegen Tonsillektomie, Adenotomie, Phimosen-, Hodenhochstand- und Hernienoperationen kamen.

Das Vergleichskollektiv der 77 gesunden Erwachsenen bestand vorwiegend aus Klinikpersonal (med. techn. Assistentinnen, Ärzte, Medizinstudenten, Küchenpersonal, Diätassistentinnen). Mit Ausnahme von Kontrazeptiva wurden keine Medikamente eingenommen. Präoperativ wurden in unserer Klinik routinemäßig folgende MeßgröBen bestimmt: kleines Blutbild mit Thrombozyten, die Konzentration von Natrium, Kalium, Harnstoff und oder Creatinin, die Aktivität der Aspartat-Aminotransferase, der Alanin-Aminotransferase und der Cholinesterase, die Thromboplastinzeit nach Quick und die aktivierte partielle Thromboplastinzeit (PTT) mit Actin ${ }^{\circledR}$-FS (Baxter).

Für die weiteren Gerinnungsuntersuchungen wurden diese Plasmen tiefgefroren bei minus $70^{\circ} \mathrm{C}$ gelagert. Die mittlere Lagerdauer betrug 16 Tage. Nach dem Auftauen wurden folgende Meßgrößen bestimmt: PTT mit Actin ${ }^{\circledR}$ FS (Fa. Baxter) und Neothromtin (Fa. Behring) dazu die Faktoren VIII und IX ebenfalls von den Behringwerken. Alle Gerinnungsuntersuchungen wurden als Doppelwerte durchgeführt und die Mittelwerte auf- bzw. abgerundet. Die CRP-Bestimmung wurde am BNA (Nephelometer der Behringwerke) durchgeführt.

In einem Begleitversuch sollte überprüft werden, ob durch Tieffrieren der Proben auf minus $70^{\circ} \mathrm{C}$ eine Veränderung der Gerinnungsaktivität zu beobachten war. Dazu wurden Proben von Kontrollplasma N (Behring Charge Nr. 502701), Pathoplasma II (Behring Charge Nr. 502954) und 10 Patientenplasmen nach Bestimmung der PTT mit Neothromtin ${ }^{\circledR}$ auf jeweils zwei Aliquots verteilt, und bei minus $70^{\circ} \mathrm{C}$ gelagert. Die PTT-Bestimmungen wurden nach einem und nach vier Monaten wiederholt; die Patientenplasmen wurden als Doppelbestimmung, die Kontrollplasmen in zwanzigfacher Bestimmung gemessen.

Das Citratblut wurde mit dem geschlossenen Blutentnahmesystem der Firma Sarstedt entnommen $(5 \mathrm{ml}$ Monovette) mit $3,13 \%$ Trinatriumcitratlösung als Antikoagulans. Das Mischungsverhältnis betrug 1:10. Die oben angegebenen Gerinnungsuntersuchungen wurden am KC 10 der Firma Amelung nach den Vorschriften der Herstellerfirma bestimmt.

Die Bestimmung der Faktoren VIII und IX erfolgte mit den Reagenzien und Mangelplasmen der Behringwerke. Bei jeder Serie wurde eine neue Eichkurve mit Standard Humanplasma erstellt. Als Kontrollen wurden für alle Gerinnungsuntersuchungen Kontrollplasma $N$ (Behring Charge Nr. 502701), Kontrollplasma P (Behring Charge Nr. 512602 A) und Pathoplasma II (Behring Charge Nr. 502954) mitgeführt. Das Blutbild wurde am Coulter Counter S Plus VI gemessen und die klinisch chemischen Bestimmungen am Hitachi 717 durchgeführt.
Die statistischen Berechnungen erfolgten nach dem Wilcoxon U-Test $(8,9,10)$ durch die Pharmaberatungsgesellschaft Kronberg/Taunus.

\section{Ergebnisse}

In dem unter Material und Methoden beschriebenen Lagerungsversuch konnten keine signifikanten Veränderungen der PTT festgestellt werden (Tab. 1).

Tab. 1: Einfluß·tiefgefrorener Lagerung auf die PTT-Bestimmung bei Kontrollproben und Patientenplasmen (Reagenz: Neothrom$\operatorname{tin}^{\infty} \%$.

\begin{tabular}{|c|c|c|c|c|c|c|c|c|c|}
\hline & \multicolumn{3}{|c|}{ Kontrollplasma $\mathbf{N}$} & \multicolumn{3}{|c|}{ Pathoplasma II } & \multicolumn{3}{|c|}{ Patientenplasmen } \\
\hline & frisch & $\begin{array}{l}1 \text { Mon } \\
-70^{\circ} \mathrm{C}\end{array}$ & $\begin{array}{l}4 \text { Mon } \\
-70^{\circ} \mathrm{C}\end{array}$ & fris & $\begin{array}{l}1 \mathrm{Mon} \\
-70^{\circ} \mathrm{C}\end{array}$ & $\begin{array}{l}4 \text { Mon } \\
-70^{\circ} \mathrm{C}\end{array}$ & fri & $\begin{array}{l}1 \mathrm{Mor} \\
-70^{\circ} \mathrm{C}\end{array}$ & $\begin{array}{l}4 \text { Mon } \\
-70^{\circ} \mathrm{C}\end{array}$ \\
\hline $\begin{array}{l}n \\
x / \\
\sec \end{array}$ & $\begin{array}{c}20 \\
29,8\end{array}$ & $\begin{array}{c}20 \\
31,4\end{array}$ & $\begin{array}{c}20 \\
30,7\end{array}$ & $\begin{array}{c}20 \\
81,0\end{array}$ & $\begin{array}{c}20 \\
81,3\end{array}$ & $\begin{array}{c}20 \\
79,5\end{array}$ & $\begin{array}{c}10 \\
29,9\end{array}$ & $\begin{array}{c}10 \\
31,2\end{array}$ & $\begin{array}{c}10 \\
32,5\end{array}$ \\
\hline
\end{tabular}

Beim Kontrollplasma $N$ wurde ausgehend von 29,8 Sekunden eine im Mittel 1,6 bzw. 0,9 längere Gerinnungszeit nach einem bzw. vier Monaten gemessen bei einem Variationskoeffizienten von 2,5 bzw 2,1\%. Beim Pathoplasma II kam es bei einem Ausgangswert von 81 Sekunden zu einer Verlängerung von 0,3 bzw. Verkürzung von 1,5 Sekunden, bei einem Variationskoeffizienten von 1,3 bzw. 1,7\%. Bei den Patientenplasmen kam es zu einer Verlängerung von 1,3 bzw. 2,6 Sekunden, ebenfalls nach einem bzw. vier Monaten. L. Smith et al. (11) kommen zu gleichen Ergebnissen. Zum Unterschied dazu wird deutlich, daß Iyophilisierte Kontrollproben tiefgefroren gelagert einen geringeren Aktivitätsverlust zeigen.

Die Variationskoeffizienten der von Tag zu Tag mitgeführten. Kontrollen betrugen über den gesamten Zeitraum (Januar 1989 bis Februar 1990) 2,7 bis 3,6\% (Neothromtin $^{\circledR}$ ) bzw. 1,8 bis $4,7 \%$ (Actin ${ }^{\circledR}$-FS) für die PTT, 7 bis $10 \%$ für den Faktor VIII und 4 bis $12 \%$ für den Faktor IX bezogen auf alle verwendeten Kontrollen. Die Variationskoeffizienten in der Serie lagen zwischen 1,3 und 2,3\% (Neothromtin ${ }^{\circledR}$ ) bzw. 0,75 und 1,0\% (Actin ${ }^{\circledR}$-FS).

Die Ergebnisse von 92 der 114 Kinder und von 69 der 77 Erwachsenen wurden ausgewertet. Die Gründe für den Ausschluß der anderen waren u. a.: erhöhte CRP-Werte, pathologisch veränderte Enzyme, erniedrigte Thrombozytenzahl, Thrombóplasstinzeit nach Quick unter $75 \%$. In Abbildung 1a sind die ermittelten PTT-Werte in Sekunden mit Neothromtin ${ }^{\circledR}$ als Einzelwerte und 5., 50., 95. Percentile bei Kindern $(30,0,34,5,43,0)$ und Erwachsenen $(27,0$, $33,0,38,0)$ aufgeführt. Im Vergleich zwischen den beiden Gruppen zeigt sich eine Verschiebung der 5 . Percentile um drei Sekunden und der 95. Percentile um fünf Sekunden nach oben bezogen auf die Erwachsenengruppe. Diese Differenzen sind statistisch signifikant ( $p<0,001$ ). Abbildung $1 b$ zeigt die ermittelten PTT-Werte mit dem Reagenz Actin ${ }^{\circledR}$-FS. Hier beträgt die Differenz zwisch»n der.Erwachsenen- $(28,0,34,0,40,0)$ und der Kindergruppe $(28,0,34,0,42,0)$ lediglich zwei Sekunden und ist statistisch nicht signifikant. Abbildung $1 \mathrm{c}$ gibt die Verhältnisse mit Frischplasma wieder. Auch hier mit dem Reagenz Actin $^{\circledR}$-FS ermittelt. Die Differenz zwischen beiden Gruppen (Erwachsene 27,0, 32,0, 39,0, Kinder 28,0, 33,0, 40,0) beträgt über den ganzen Bereich zwar lediglich eine Sekunde, ist aber statistisch signifikant $(p<0,05)$. 
$\mathrm{PTT}(\mathrm{sec})$

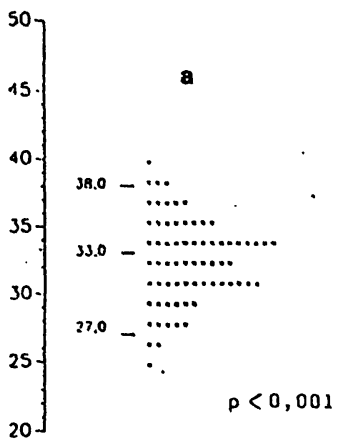

.

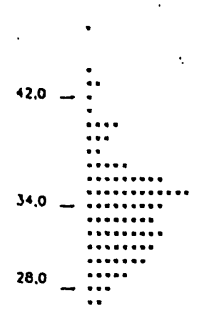

Die Abbildungen 2 und 3 geben die Einzelwerte und die 5., 50. und 95. Percentile bei Erwachsenen und Kindern für die Faktoren VIII und IX wieder. Beim Faktor VIII liegt bei den Erwachsenen die 50 . Percentile bei $92,3 \%$, bei den Kindern bei 109,3\%. Während die obere Grenze 195 . Percentile) in beiden Gruppen gleich ist $(160,3 \%)$, unterscheidet sich die untere Grenze ( 5 . Percentile) in beiden Kollektiven um 8,5\%.

Beim Faktor IX liegt die 50. Percentile bei $85 \%$ (Erwachsene) bzw. $77 \%$ (Kinder). Der Bereich erstreckt sich von 60 bis $120 \%$ (Erwachsene) und 60 bis $98 \%$ (Kinder).

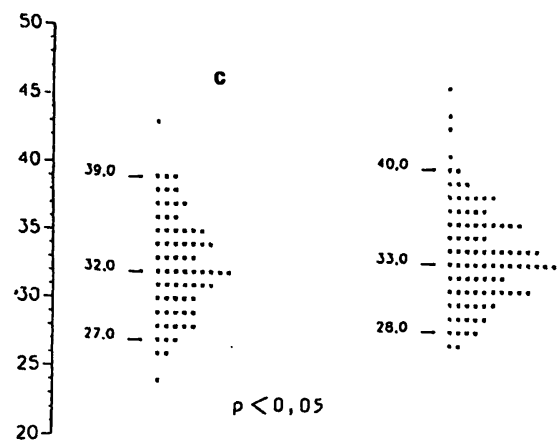

$$
\begin{array}{cc}
\text { Erwochserie } & \text { Kinder } \\
(n=69) & (n=92)
\end{array}
$$

Abb. 1: Vergleich der PTT-Werte zwischen Kindern und Erwachsenen mit Angabe des Medians und der 5. und 95. Percentile.

a: Reagenz Neothromtin ${ }^{\circledR}$, tiefgefrorene Plasmen

b: Reagenz Actin ${ }^{\circledR}$-FS, tiefgefrorene Plasmen

c: Reagenz Actin ${ }^{\circledR}$-FS, Frischplasmen

In der Abbildung 4 wurden auf der X-Achse die prozentualen Werte der gemessenen Faktoren VIII und IX gegen die PTT-Werte (Neothromtin ${ }^{\circledR}$ ) in Sekunden auf der $Y$ Achse aufgetragen. Das eingezeichnete Fenster zeigt die 5. 'bis 95. Percentile von PTT und Faktor VIII (4a) und Faktor IX (4b). Bei drei Kindern wurden PTT-Werte über 43 Sekunden gemessen, trotz normaler Faktor VIII-Aktivität (Abb. 4a), bei zwei Kindern fanden sich Faktor VIII-Aktivitäten unterhalb der 5 . Percentile von $76,5 \%$ bei normalen PTT-Werten. In der Erwachsenengruppe findet man zwei Erwachsene mit normaler PTT und erniedrigtem Faktor VIII und einen mit verlängerter PTT (über 38,0 Sekunden)

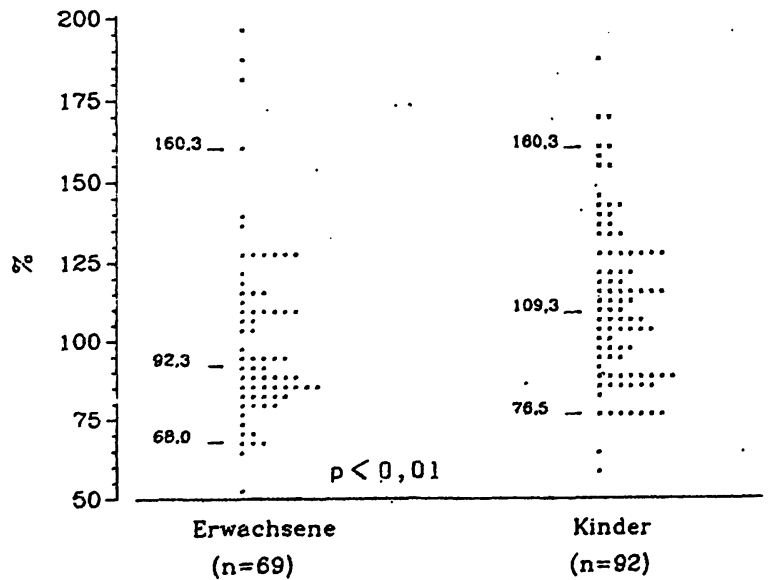

Abb. 2: Faktor VIII-Werte bei Kindern und Erwachsenen mit Angabe des Medians und der 5. und 95. Percentile.

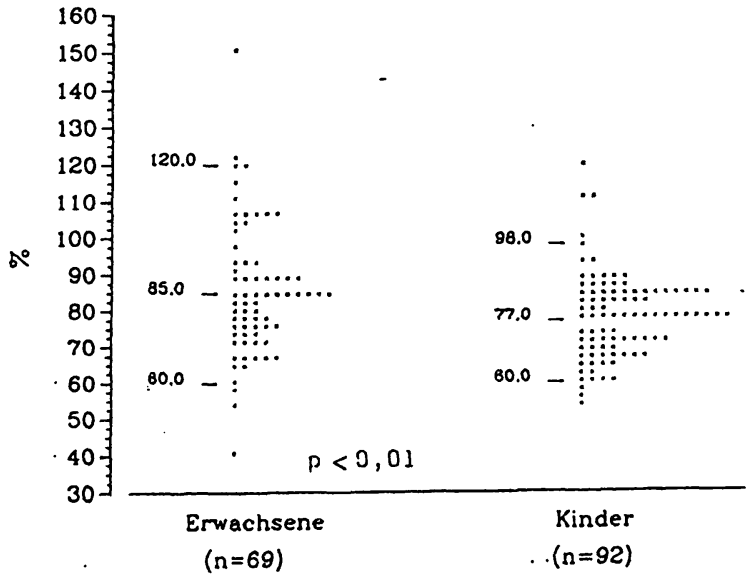

Abb. 3: Faktor IX-Werte bei Kindern und Erwachsenen mit Angabe des Medians und der 5. und 95. Percentile. 
und normaler Faktor VIII-Aktivität. In Abbildung $4 b$ hatten zwei Kinder ein erhöhte PTT und erniedrigte Faktor IX-Aktivität, ein Kind hatte eine normale PTT bei leicht erniedrigtem Faktor IX und ein weiteres Kind hatte eine verlängerte PTT bei grenzwertig erniedrigter Faktor IX-Aktivität. Bei den Erwachsenen fanden sich einer mit erhöhter PTT und normaler Faktor IX-Aktivität und weitere drei mit normaler PTT und erniedrigtem Faktor IX.

\section{Diskussion}

Jeder, der sich mit der Erstellung von Richtwerten im Kindesalter beschäftigt, weiß um die Schwierigkeiten, ein ausreichend großes Kollektiv gesunder Kinder verschiedener Altersstufen zu erhalten. Im Kindesalter gibt es keine Situation, in der bei routinemäßigen Reihenuntersuchungen auch Blut abgenommen wird, um Referenzwerte zu erstellen. Bei Kindern wird angesichts der Problematik der ausreichenden Probengewinnung über das absolut Notwendige hinaus darauf verzichtet. Da jedoch keine Informationen über die Aktivität des Gerinnungssy- stems im Vorschul- und Schulalter vorliegen, wurden Untersuchungen an Kindern durchgeführt, deren Erkrankungen keinen Einfluß auf das Gerinnungssystem haben und wo wegen des beabsichtigten Eingriffes eine Blutentnahme unumgänglich war.

Die erzielten Ergebnisse bestätigen die in der Einleitung beschriebenen klinischen Erfahrungen. Vergleicht man die mit Neothromtin gemessenen PTT-Werte (Abb. 1) des Erwachsenen-Kollektivs mit dem Kollektiv der Kinder, so findet sich eine statistisch gesicherte Differenz von 5 Sekunden im Bereich der 95. Percentile. Hingegen ist die Differenz zwischen Erwachsenen und Kindern bei den PTT-Messungen mit dem Reagenz Actin -FS nur im Frischplasma, aber nicht im tiefgefrorenen Plasma statistisch signifikant unterschiedlich. Die Lagerung bei minus $70^{\circ} \mathrm{C}$ führt beim Actin ${ }^{\circ}$-FS zu etwas längeren Zeiten und verwischt diesen Unterschied so, daß er statistisch nicht mehr signifikant ist. Der in Tabelle 1 bei Patientenplasmen mit Neothromtin gefundene Unterschied entspricht weitgehend dem von Actin ${ }^{\otimes}$-FS zwischen frischen und tiefgefrorenen Proben. Beide Reagenzien enthalten
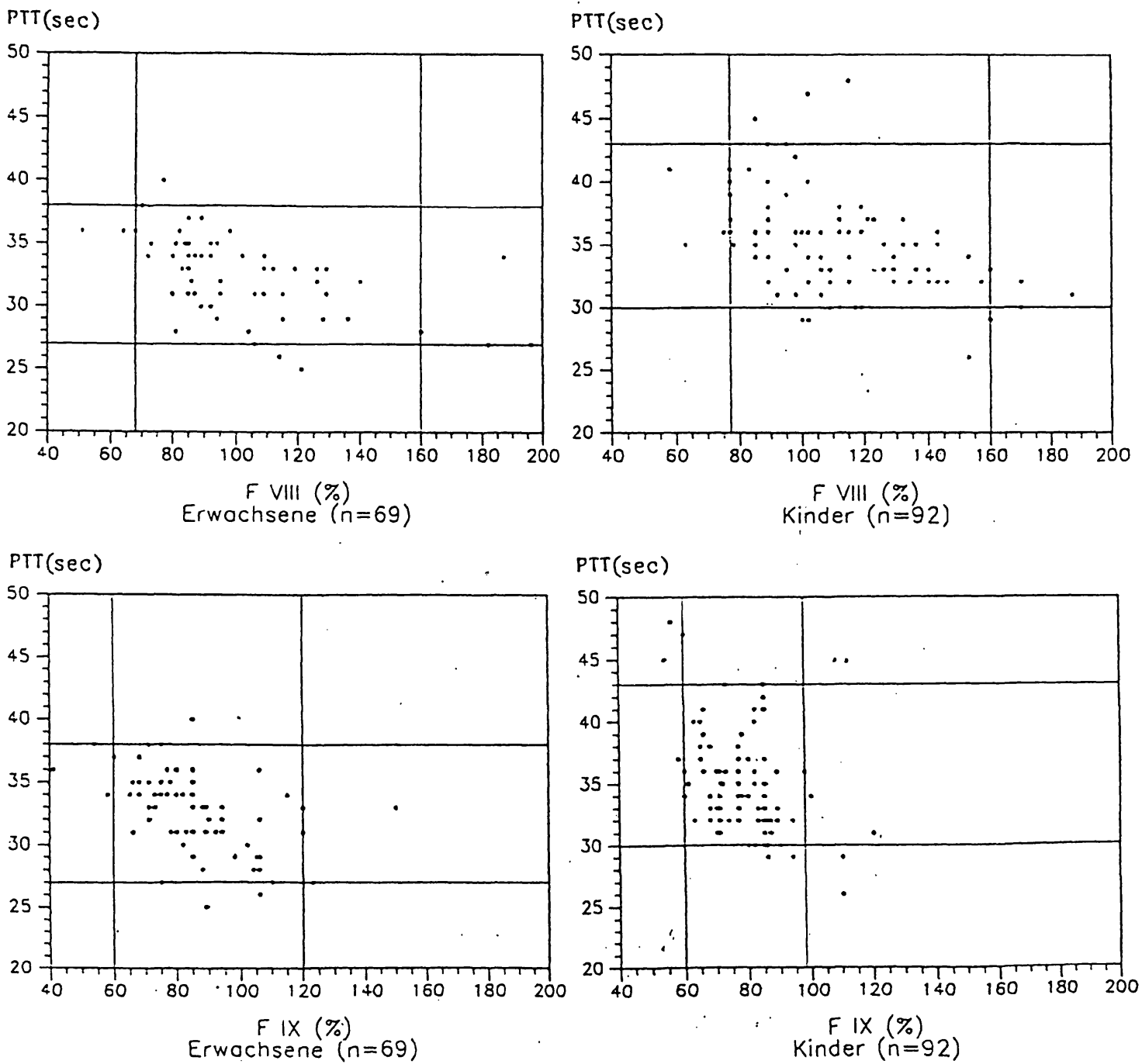

Abb. 4 a u. b: Darstellung der PTT-Werte in Sekunden gemessen mit Neothromtin ${ }^{\circledR}$ und der Faktor VIII- und IX-Aktivitäten in Prozent bei Kindern und Erwachsenen unter Angabe der 5. und 95. Percentile. 
als Aktivator gemeinsam die Ellagsäure und ein pflanzliches Phospholipid aus Sojabohnen, jedoch dürften die Stabilisatoren, die verwendeten Pufferlösungen und die Kalziumkonzentrationen unterschiedlich sein, was möglicherweise zu den oben erwähnten Differenzen geführt hat $(12,13)$.

Die Sensitivität gegenüber den Faktoren VIII und IX ist bei beiden Reagenzien gleich gut und höher als bei Reagenzien, die mit Kaolin oder Silicataktivierung arbeiten $(11,13,14,15)$ und mit denen in den Jahren vor 1970 Normalwerte für die PTT ermittelt wurden.

Für das Actin ${ }^{\circledR}$-FS wurden die von der Firma angegebenen Richtwerte für Frischplasmen (bis 40 Sekunden) sowohl für Erwachsene als auch für Kinder bestätigt. In tiefgefrorenen Proben fand sich eine Anhebung der 95. Percentile um zwei Sekunden. Mit dem Reagenz Neothrom$\operatorname{tin}^{\circledR}$ konnten nur Plasmen nach vorheriger Lagerung bei minus $70^{\circ} \mathrm{C}$ bestimmt werden. Dadurch fand sich erwartungsgemäß, wie schon Tabelle 1 deutlich macht, eine Verschiebung der von der Firma angegebenen oberen Grenze von 35 Sekunden auf 38 Sekunden.

Die angegebenen Richtwerte sollten mit den in der Klinik üblichen Abnahme- und Bestimmungstechniken überprüft werden, da große Streuungen beschrieben wurden $(11,12,14,15,16)$.

Die ermittelten 5., 50. und 95. Percentile für die Aktivitäten der Faktoren VIII und IX entsprechen weitgehend den Angaben anderer Untersucher $(2,3,17)$. Auch bei der $\mathrm{Ge}-$ genüberstellung von Faktorenaktivität zu PTT-Wert liegen sowohl Kinder als auch Erwachsene, von den zu erwartenden Ausnahmen abgesehen, innerhalb des angegebenen Bereiches (Abb. 2, 3, 4). Aufgrund der geringen Blutmenge mußte die Bestimmung des Faktors XII entfallen.

Eine mögliche Ursache für die gefundene Differenz zwischen Kinder- und Erwachsenenkollektiv könnte das unterschiedlich häufige Auftreten von Lupusantikoagulanz. sein. Diese Antikörper, die gegen Phosphatidylserin bzw. Phosphatidyläthanolamin gerichtet sind, treten bei Kleinkindern vorübergehend postinfektiös besonders bei Tonsillitiden auf (18). Obwohl nur Kinder mit normalem CRP in die Studie aufgenommen wurden und im entzündungsfreien Intervall operiert wurden, kann nicht völlig ausgeschlossen werden, daß einzelne Kinder mit Lupusantikoagulanz in der Studie enthalten sind. Die in Lit. (19) beschriebenen Fälle von Lupusantikoagulanz, die alle eine Hypoprothrombinämie aufwiesen, wären nicht in die Studie eingegangen, da Quickwerte unter $75 \%$ als Ausschlußkriterium galten.

Bei den 92 Kindern ergeben sich unter Berücksichtigung ihrer 95. Percentile zwei grenzwertige und drei erhöhte PTT-Werte, wobei alle Faktor VIII-Werte im Normbereich liegen, während Faktor IX einmal grenzwertig und in zwei Fällen erniedrigt gefunden wird. Unter Zugrundelegung der 95. Percentile der Erwachsenen würden zusätzlich 9 der 92 Kinder erhöhte PTT-Werte aufweisen, bei denen bis auf einen niedrigen Faktor VIII-Wert alle Faktor VIIIund Faktor IX-Aktivitäten im Normbereich liegen.

Für die routinemäßige präoperative Screening-Situation mit Hilfe der PTT bedeutet dies, daß bei eigenen KinderReferenzwerten $3 \%$ der Kinder erhöhte PTT-Werte aufweisen, wobei $2 \%$ auffallend niedrige Faktoren-Aktivitäten haben. In keinem Fall jedoch waren klinisch relevante Gerinnungsstörungen $\mathrm{zu}$ beobachten. Ohne eigenen Normbereich würden dagegen $15 \%$ der Kinder erhöhte PTT-Werte aufweisen, wobei nur bei $3 \%$ auffallend niedrige Faktoren-Aktivitäten vorliegen. Aus dieser Konstella- tion ergibt sich zwingend die Notwendigkeit, eigene Richtwerte für Kinder im Alter von zwei bis zehn Jahren zu erstellen.

\section{Danksagung}

Für die họhe Kooperativität und die Überlassung der Plasmen und der klinischen Daten sind wir dem Leiter der Kinderklinik des Harlachinger Krankenhauses, Prof. Dr. med. Klaus Dieter Tympner, dem OA Dr. med. F. Snigula und den Mitarbeitern der Stationen K4 und K6 zu besonderem Dank verpflichtet. Frau G. Plitt (Behringwerke AG) danken wir für die Unterstützung bei der Literaturrecherche.

Schrifttum:

1. Struckmeyer, H., Haid, H. (1986) Richtwerte für das kinderärztliche Laboratorium. Die medizinische Verlagsgesellschaft $\mathrm{mbH}$, Marburg, 38

2. Wallach, J. (1983) Interpretation of Pediatric Tests. Little, Brown \& Company, 9. 3. Kreuz, W. D., Nowak-Göttl, U., Krackhardt, B., Hach-Wunderle, V. \& Scharrer, I. (1989) Platelet count, von Willebrand factor, factor Villc, ristocetin cofactor, Protein $C$ and antithrombin III in 74 healthy children (a control group). Blut 58, 101.

4. Mingers, A. M., Stroeder, J. (1980) Einfluß hypokalorischer Kost auf die Blutgerinnung übergewichtiger Kinder. Monatsschr. Kinderheilkunde 128, 558-562.

5. Stroeder, J., Mingers, A. M. (1979) Gerinnungsstudien bei adipösen Kindern unter Reduktionsdiät. Monatsschr. Kinderheilkunde 127, 265-268.

6. Andrew, M., Paes, B., Milner, R., Johnston, M., Mitchell, L., Tollefsen, D. M. \& Pow6. Andrew, M., Paes, B., Milner, R., Johnston, M., Mitchell, L., Tollefsen, D. M. \& Pow-
ers, P. (1987) Development of the human coagulation system in the Full-Term infant. Blood 70, 1, 165-172.

7. Andrew, M., Paes, B., Milner, R., Johnston, M., Mitchell, L., Tollefsen, D. M., Castle, V. \& Powers, P. (1988) Development of the human coagulation system in the healthy premature infant. Blood 72, 5, 1651-1657.

8. Wilcoxon, F. (1945) Individual comparisons by ranking methods. Biometrics Bulletin $1,6,80-83$.

9. Wilcoxon, F. (1946) Individual comparisons of grouped data by ranking methods. Journal of Economic Entomology 39, 2, 269-272.

10. Wilcoxon, F. (1947) Probability tables for individual comparisons by ranking methods. Biometrics Bulletin, 3, 3, 119-122.

11. Smith, L. G. \& Kitchens, G. S. (1985) A comparison between two commercially available activators for determining the partial thromboplastin time. Arch. Pathol. Lab. Med. 109, 243-246.

12. Marlar, R. A., Bauer, P. J., Endres Brooks, J. L., Montgomery, R. R., Miller, Ch. M. \& Schanen, M. M. (1984) Comparison of the sensitivity of commercial APTT reagents in detection of mild coagulopathies. Am. J. Clin. Pathol. 82, 4, 436-439.

13. Schwinn, H. Herchenhan, B. (1980) Ein optisch klares Reagenz zur Bestimmung 13. Schwinn, H., Herchenhan, B. (1980) Ein optisch klares Reagenz zur Bestimmung der aktivierten Partiellen Thromplastin-Zeit, Fibrinolys
Deutsch, E., Lechner, K. F. K., Schattauer Verlag, 411-413.

14. Baumann, L. \& Zimmermann, T. S. (1985) Partial Thromboplastin Time reagents: Another look. Lab.med. 16, 1, 35-37.

15. Turi, D. \& Peerschke, E. (1986) Sensitivity of three activated thromboplastin time reagents to coagulation factor deficiencies. Am. J. Clin. Pathol. 85, 1, 43-49.

16. O'Brien, P. F., North, W. R. S.\& Ingram, G. I. C. (1981) The diagnosis of mild haemophilia by the partial thromboplastin time test WFH/ICTH study of Manchester method. Thrombos. Haemostas. 45, 2, 162-168.

Thrombos. Haemostas. 45, 2, 162-168.
17. Tietz, N. W. (1983) Clinical Guide to laboratory tests. W. B. Saunders Comp.

18. Mingers, A. M., Sutor, A. H. \& Kreth, H. W. (1991) The significance of lupus anticoagulants in childhood. Annals of Hematology. Abstract, 36 .

19. Jäger, U. \& Kapiotis, S. (1991) Postinfectious lupus anticoagulants. Annals of Hematology. Abstract, 81.

Anschrift der Verfasser:

Dr. med. Gisela Wanner

Prof. Dr. med. Ludwig Weiss

Dr. Janny van der Woerd-de Lange

Institut für Klinische Chemie am Städtischen Krankenhaus

München-Harlaching

Sanatoriumsplatz 2

8000 München 90

Dr. med. Norbert Hegner

Behringwerke AG

Medizinische Information und Verkauf

Kurmainzerstraße 6

6230 Frankfurt am Main 80 